\title{
ARTICLE
}

\section{Design of a new integral benchmark experiment for large angle scattering using shadow bar and DT neutron source}

\author{
Seiki Ohnishi $^{\mathrm{a}^{*}}$ and Isao Murata ${ }^{\mathrm{b}}$ \\ ${ }^{a}$ National Maritime Research Institute, 6-38-1 Shinkawa, Mitaka-si, Tokyo, 181-0004, Japan; ${ }^{b}$ Osaka University, 1-1 Yamadaoka, \\ Suita-si, Osaka 565-0871, Japan
}

\begin{abstract}
A new integral benchmark experiment was designed for validating large angle scattering cross section. In conventional integral benchmark experiments, events due to small angle scattering was dominant and the information on large angles ones were very little. Therefore, we designed a new experiment using a shadow-bar to suppress the contribution of small angle scatterings and direct incidents from the neutron source. The geometry of the experiment consists of a DT (Deuteron-Triton fusion) neutron source, a shadow-bar, a neutron detector and a target plate. To search the optimum configuration in which large angle scatterings were counted more frequently, the neutron transport was simulated with altering the target plate angle and the position of the neutron detector. It turned out that in the case where the target plate was set at a slant against the shadow-bar, the average scattering angles of the tallied neutrons became smaller. If the activation foil was set on the edge of the target plate, the contribution of large angle scatterings and the reaction rate of the activation foil decreased. By choosing these parameters properly, this new designed experiment may enable large angle scattering cross sections to be validated.
\end{abstract}

Keywords: integral benchmark; scattering cross section; Monte Carlo method; MCNP; activation foil; shadow-bar

\section{Introduction}

Many integral experiments using deuteron-triton fusion (DT) neutrons, for example in sphere [1,2], cube $[3,4]$ or cylindrical assembly [5], have been conducted. In those experiments, small angle elastic scattering events are dominant because its cross section is larger than that of large angle scattering. In order to validate large angle scattering cross section, we started to design a new integral experiment with a DT neutron source. For this purpose, it is required to prevent small angle scattered neutrons from contributing to neutron detectors.

If the contributions of large angle scatterings are measured by comparing several experiments' results with several calculation results using different nuclear libraries, it may be possible to discuss the controversial angular region of the scattering cross sections. The aim of this work is to design a new integral benchmark experiment in which the contributions of small angle scatterings are reduced. In section 2, the experimental geometry design is described and the characteristics of this experiment is analyzed and discussed in section 3. Finally, this paper is concluded in section 4.

*Corresponding author. Email: ohnishi@nmri.go.jp

\section{Geometry design}

\subsection{Component}

To reduce the contribution of small angle scattering is the most important for the new benchmark experiment. Moreover, it is required to reduce source neutrons directly incident on the detector. To satisfy these requirements, a shadow-bar experiment is introduced. The geometry of this new experiment consists of a DT neutron source, a shadow-bar, a target plate and a small neutron detector. The shadow-bar was laid between the detector and DT neutron source to shield the direct neutron contribution to the detector. Consequently, the neutrons entered the detector after a few times scattering within the target plate. The basic geometry is shown in Figure 1.

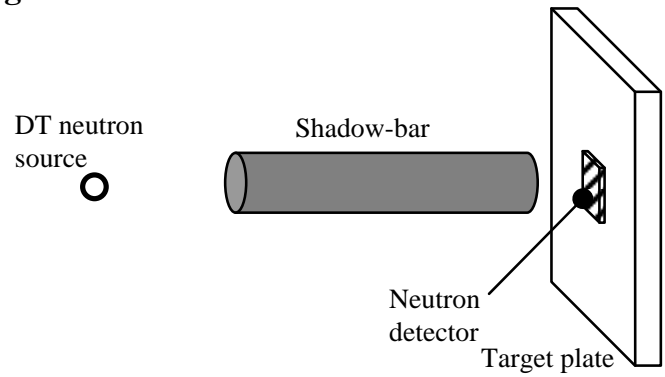

Figure 1. The basic geometry of this new experiment. 
This method has an advantage that a relatively large target can be used and more frequent scatterings are expected to occur. However, the effect of small angle scattering will be more enhanced if the target plate becomes too thick. Therefore, the thickness of the target was limited to less than a few mean-free-paths.

\subsection{Configuration parameters}

Figure 1 shows the basic components of the experiment setup. However two parameters still remained. One was the position where the neutron detector was set on and another was the angle of the target plate against the shadow-bar. We considered two types of the detector position; one was setting the detector on the center of the target plate and the other was on the edge. As to target angles, four angles, -22.5, 0, 22.5 and 45 degrees, were considered.

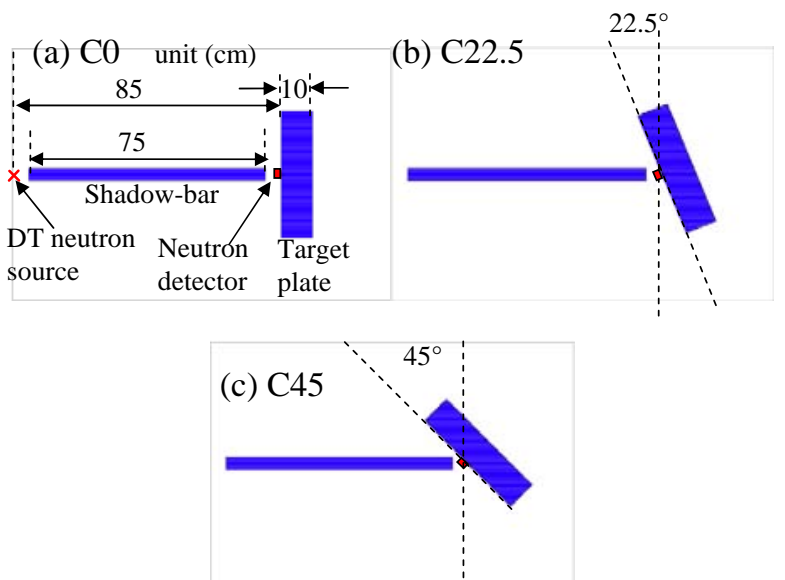

Figure 2. Detector centered configurations. (a) E0

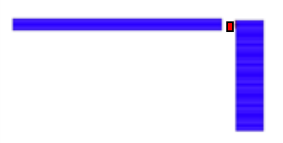

(c) E45

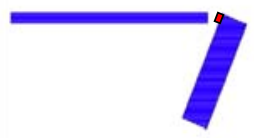

(d) E-22.5

(b) E22.5

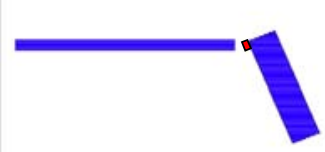

Figure 3. Detector off-centered configurations. (a)E0, (b)E22.5, (c) E45 and (d) E-22.5.

These configurations were named as below by their detector position and target angle; (1) "C0" represents that the detector was set on the "center" of the plate and the angle between shadow-bar and target plate was " 0 " degree, (2) “C22.5” represents center and 22.5 degrees, (3) "C45" does center and 45 degrees, (4) "E0" does edge and 0 degree, (5) “E22.5” does edge and 22.5 degrees, (6) "E45" does edge and 45 degrees and (7) "E-22.5" does edge and minus 22.5 degrees. It is remarked that C-22.5 and C22.5 is the same because of symmetric property and the former was omitted. These configuration patterns are shown in Figures $\mathbf{2}$ and $\mathbf{3}$. The influence of the target angle and the detector position was analyzed in the next section.

\section{Collision histograms in new experiments}

\subsection{Analyzing method}

To validate large angle scattering cross section, we should choose configurations in which large angle scatterings occur frequently and enough number of counts can be acquired. To clarify which angle of scattering is frequent and to estimate the detection efficiency in these configurations, the neutron transportation was simulated and the tracks of neutrons were analyzed. In this simulation, the Monte Carlo transport calculation code, MCNP5 [6], and evaluated nuclear data library, JENDL-4 [7] were used and then all the neutrons' tracks and events were recorded.

As the typical cases, the length, width, and thickness of the target plate were set to be $40 \mathrm{~cm}, 40 \mathrm{~cm}$ and $10 \mathrm{~cm}$ respectively. The shadow-bar and the target plate were made by iron. The cylinder shape shadow-bar should have enough length to shield the neutrons which enter the detector directly from the DT neutron source. The length of the shadow-bar was set to be $75 \mathrm{~cm}$, longer than ten times of the mean-free-path of DT neutrons in iron. This study is focused on the angular scattering cross sections, therefore to simplify the analyses, the energy region for validation was restricted to DT neutron energy; i.e. around $14 \mathrm{MeV}$. It is necessary to note that the elastic and inelastic cross section of Fe-56 at $14 \mathrm{MeV}$ are 1.199 barn and 0.773 barn respectively [7] and consequently, the validation will be conducted against the sum of elastic and inelastic cross sections.

For the purpose of this simplification, a piece of niobium foil was adopted as a neutron detector and its width, length and thickness is $3 \mathrm{~cm}, 3 \mathrm{~cm}$ and $0.2 \mathrm{~cm}$. This size of track-length estimator was set on the target plate and the ${ }^{93} \mathrm{Nb}(\mathrm{n}, 2 \mathrm{n}){ }^{92 \mathrm{~m}} \mathrm{Nb}$ reaction was tallied. Thus the reaction rate of the activation foil was calculated and the collision histograms depending on scattering angle were made by keeping the tracks of tallied neutrons.

\subsection{The influence of detector position}

Figures 4, 5 and 6 are the scattering angle histograms. The origins of scattering angle in these analyses were set at the direction of neutrons movement before their neutron-nucleus collisions. These figures show that the position of the detector on the target plate makes little difference. 


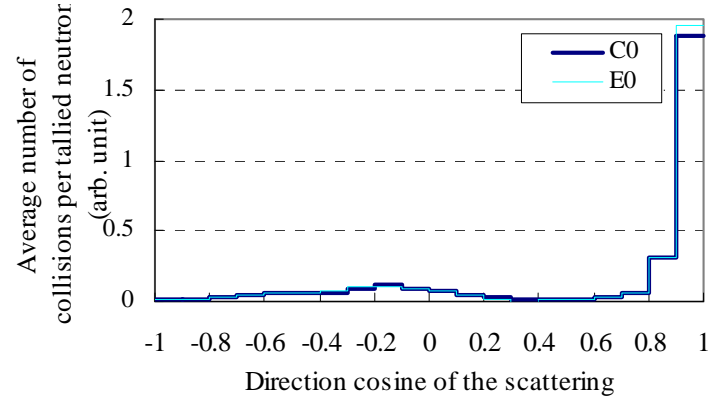

Figure 4. Collision histograms of C0 and E0.

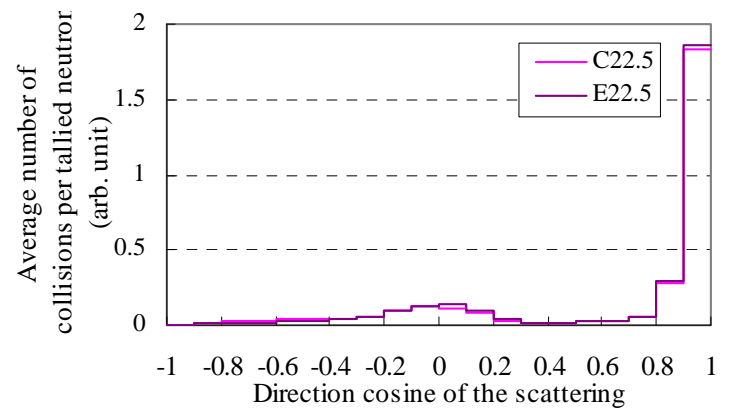

Figure 5. Collision histograms of C22.5 and E22.5.

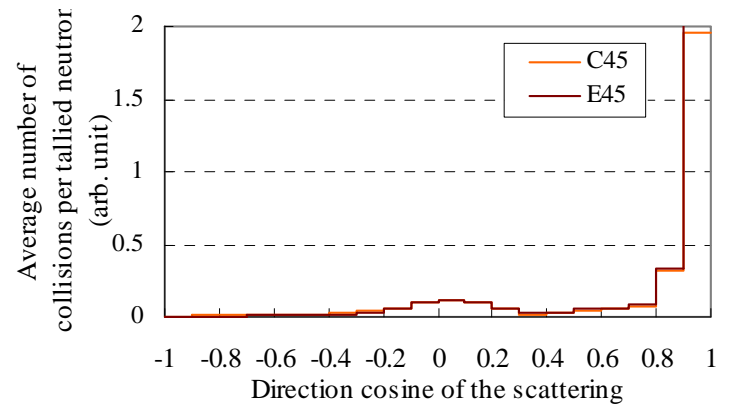

Figure 6. Collision histograms of C45 and E45.

While the shadow-bar was set and the direct incident neutrons were shielded, the small angle scattering were dominant in all the cases. Figure 7 plots the points where the scattering reactions occurred by the "tallied" neutrons in $\mathrm{C} 0$ configuration.

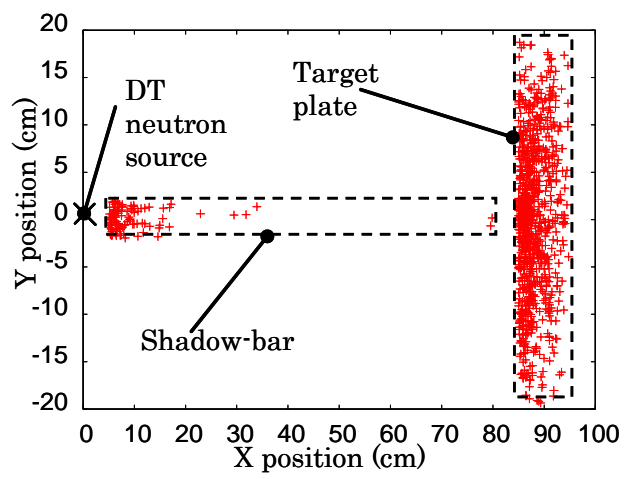

Figure 7. 1,000 collision locations in C0 configuration. The origin is located at the DT neutron source.
The collision points of the tallied neutrons distribute almost uniformly in the target plate even behind the shadow-bar. This shows that multiple scatterings occur before the neutrons enter the detector. This is because the target is thick enough to allow neutron multiple scattering within the target plate.

In the detector-off-centered configuration, the frequency of small angle scattering becomes slightly larger than in detector-centered configuration. In all the cases, the small angle scattering is dominant because its cross section is larger than that of large angle scattering and the thickness of the target is much larger than one mean-free-path of DT neutrons in iron.

Table 1. Reaction rates of the activation foil.

\begin{tabular}{lcc}
\hline $\begin{array}{l}\text { Position of } \\
\text { activation foil }\end{array}$ & $\begin{array}{l}\text { Target angle } \\
\text { (degrees) }\end{array}$ & $\begin{array}{l}\text { Reaction rate } \\
\text { (reaction/source) }\end{array}$ \\
\hline C0 & 0 & $6.22 \times 10^{-33}$ \\
C22.5 & 22.5 & $7.96 \times 10^{-33}$ \\
C45 & 45 & $7.87 \times 10^{-33}$ \\
\hline E0 & 0 & $2.24 \times 10^{-33}$ \\
E22.5 & 22.5 & $4.22 \times 10^{-33}$ \\
E45 & 45 & $4.95 \times 10^{-33}$ \\
E-22.5 & -22.5 & $1.49 \times 10^{-33}$ \\
\hline
\end{tabular}

Table 1 shows the reaction rates of the ${ }^{93} \mathrm{Nb}(\mathrm{n}, 2 \mathrm{n}){ }^{92 \mathrm{~m}} \mathrm{Nb}$ reaction in each configuration. The reaction rate in $\mathrm{C} 0$ is almost three times as large as that of $\mathrm{E} 0$, and the reaction rates in $\mathrm{C} 22.5$ and $\mathrm{C} 45$ rates are twice larger than in E22.5 and E45. The detector-centered configuration is clearly superior in the view of detection efficiency. There is a trade-off between the frequency of large angle scattering and the reaction rate of the activation foil. Nevertheless, assuming the efficiency of detector for counting photon of the activation foil is $2 \%$, source neutron intensity is $10^{9}$ neutron/s, irradiation duration is 8 hours and measuring time of the activation foil is 24 hours, the photo-electric peak counts become about 100 . Therefore these configurations are marginal.

\subsection{The influence of target plate angle}

Figure 8 is the histograms grouped by the position of the detector. All the histograms have two peaks, i.e., the larger one around 0 degree direction and the other small one around 90 degrees. The scattering angle corresponding to the small peaks varies according to the angle of the target plate and do not depend on the position of the detector. Furthermore, the larger the angle of target becomes, this small peak become closer to the 0 degree direction. It should be noticed that the small peak exists around rather large angles in E-22.5 configuration although the detection efficiency is low as described in the previous subsections. It should be noticed that the scale of y-axis of this figure is the same as Figures 5 and 6.

To compare the efficiency how often the large angle scatterings occur, the scales of $\mathrm{x}$-axes in these histograms are simplified to only two intervals corresponding to small angle and large angle; (1) direction cosine is 

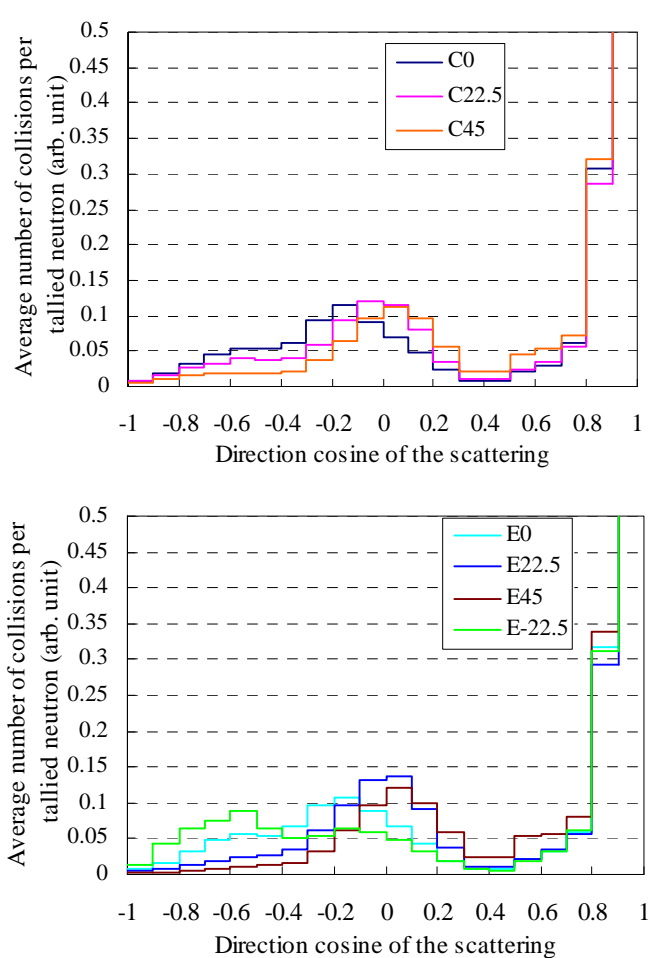

Figure 8. Collision histograms grouped by the detector position.

over 0.6 and (2) is under 0.6. Table 2 summarizes the ratios of small angle and large angle scattering frequency of the tallied neutrons in each configuration. It is clarified that large angle scatterings occurred more frequently in the detector-centered configurations.

Table 2. Ratios of the large angle and small angle scattering frequency.

\begin{tabular}{lc}
\hline Configuration & $\begin{array}{c}\text { Large angle } \\
\text { /Small angle }\end{array}$ \\
\hline C0 & 0.33 \\
C22.5 & 0.34 \\
C45 & 0.28 \\
\hline E0 & 0.31 \\
E22.5 & 0.32 \\
E45 & 0.25 \\
E-22.5 & 0.30 \\
\hline
\end{tabular}

\section{Conclusion}

The concept of the new integral experiment has been presented. By analyzing particle tracks, it is clarified which angle of scattering is occurring and contributing to the detector in the seven different configurations. In detector-centered configurations, the large angle scatterings occurred more frequently and detection efficiencies became larger than in the detector-off-centered configurations if the target angles are the same. In the configuration where the target plate was set at a slant against shadow-bar, the scattering angles of the tallied neutrons become closer to 0 degree direction. Certainly, the contribution of small angle scattering is dominant in all the configurations, however, small angle scattering cross section has been validated in past experiments and is considered valid enough. Because the contributions of large angle scattering vary according to configurations, it is expected that the data in different configuration will enable to validate scattering cross sections data in different angular range. Therefore, the validation of large angle scattering cross section will be possible by comparing the ratios' between experiments and calculations.

\section{References}

[1] C. Ichihara, S. A. Hayashi, I. Kimura, J. Yamamoto and A. Takahashi, Measurement of leakage neutron spectra from a spherical pile of niobium bombarded with $14 \mathrm{MeV}$ neutrons and validation of its nuclear data, J. Nucl. Sci. Technol. 38 (2001), pp.959-966.

[2] K. Sumita, A. Takahashi, H. Hashikura, Y. Oka and S. An, Measurements of Neutron Leakage Spectra from $50.32 \mathrm{~cm}$ Radius Iron Sphere, OKTAVIAN Report A-83-07, Osaka University, (1983).

[3] K. Ochiai, K. Kondo, S. Ohnishi, K. Takakura, S. Sato, Y. Abe, C. Suzuki, T. Yagi and C. Konno, DT neutronics benchmark experiment on lead at JAEA-FNS, Journal of the Korean Physical Society 59-2 (2011), pp.1953-1956.

[4] F. Maekawa, Y. Kasugai, C. Konno, I. Murata, Kokooo, M. Wada, Y. Oyama, Y. Ikeda and A. Takahashi, Benchmark experiment on vanadium with D-T neutrons and validation of evaluated nuclear data libraries by analysis of the experiment, J. Nucl. Sci. Technol. 36-3 (1999), pp.242-249.

[5] H. Maekawa, Y. Ikeda, Y. Oyama, S. Yamaguchi, K. Tsuda, T. Fukumoto, K. Kosako, M. Yosizawa and T. Nakamura, Fusion Blanket Benchmark Experiments on a $60 \mathrm{~cm}$-Thick Lithium Oxide Cylindrical Assembly, JAERI-M 86-182, Japan Atomic Energy Research Institute, (1986).

[6] X-5 Monte Carlo Team, MCNP - A General Monte Carlo N-Particle Transport Code, Version 5, LA-UR-03-1987, Los Alamos National Laboratory, (2003).

[7] K. Shibata, O. Iwamoto, T. Nakagawa, N. Iwamoto, A. Ichihara, S. Kunieda, S. Chiba, K. Furutaka, N. Otuka, T. Ohsawa, T. Murata, H. Matsunobu, A. Zukeran, S. Kamada and J. Katakura, JENDL-4.0: A new library for nuclear science and engineering, $J$. Nucl. Sci. Technol. 48-1 (2011), pp.1-30. 